\title{
Research on the Design of E-coupons for Directional Marketing of Two Businesses in Competitive Environment
}

\author{
Xiong Keyi ${ }^{1}$, Yang Wensheng ${ }^{2}$ \\ ${ }^{1}$ High School, Nanjing Foreign Language School, Nanjing, PR China \\ ${ }^{2}$ School of Economics \& Management, Nanjing University of Science \& Technology, Nanjing, PR China \\ Email address: \\ ritaxiong622@126.com (Xiong Keyi),wensheng_yang@163.com (Yang Wensheng)
}

\section{To cite this article:}

Xiong Keyi, Yang Wensheng. Research on the Design of E-coupons for Directional Marketing of Two Businesses in Competitive Environment. International Journal of Economics, Finance and Management Sciences. Vol. 8, No. 1, 2020, pp. 49-56. doi: 10.11648/j.ijefm.20200801.16

Received: February 2, 2020; Accepted: March 6, 2020; Published: March 10, 2020

\begin{abstract}
With the rapid development of information technology, e-coupons have shown an explosive trend in recent years. One of the biggest benefits of e-coupons is that they can achieve precise marketing, that is, they can push different coupons to different customers. Although the research on coupons has always been a hot topic, the research on e-coupons has gained increasing attention in recent years. With scant attention devoted to the research on the design of coupons for directional marketing, we investigate the design of coupon for two businesses under the competitive environment. For the old customers, the threshold reduction coupons are used, and for the new customers, the instant reduction coupons are used. Aiming at the maximization of the profits of the merchants, a coupon model for new and old customers of two merchants is built in the competitive market environment with Stackelberg games. Finally, taking two merchants with different profit as an example, the influence of competitors on the coupon delivery strategy of merchants is analyzed by Matlab program. The results show that the existence of competitors will force the merchants to further let customer and enlarge the coupon's face value to the maximum value they can bear. Overall, the study sheds light on design of e-coupons under competition and provides managers with a blueprint to improve profits by targeting shoppers with customized coupons.
\end{abstract}

Keywords: Consumer Behavior, Targeted Marketing, E-coupon, Launch Strategy, Game Theory

\section{Introduction}

With the rapid development of information technology, network has become an important channel for many businesses to carry out marketing activities. By December 2018, the online customer in China has reached 610 million, which is $14.4 \%$ higher than 2017 and accounting for $73.6 \%$ of the total Internet users [1]. Coupons are a very popular form of promotion in online shopping. In many e-commerce platforms, some coupons are often issued to attract consumers.

As an important promotion tool of Internet marketing, e-coupons have shown an explosive trend in recent years. Compared with paper coupons, electronic coupons have the characteristics of wide spread, fast spread and low spread cost. Besides, they also have the greatest advantage of precision marketing, that is, they can push different coupons to different customers and ensure that the coupons they get are what they need. At this time, our coupons become "guided" coupons, which can guide users to the direction we want to develop, and bring a very obvious promotion effect to businesses. The statistics [2] show that $40 \%$ of Americans and 50\% of French will look for coupons for online shopping, compared with $70 \%$ and $60 \%$ in Italy and Spain. However, businesses in China often face the situation that e-coupons are not attractive enough and the utilization rate is not high. Moreover, if the coupon setting is not reasonable enough, it may lead to loss or even bankruptcy of the merchants [3]. Therefore, how to reasonably develop e-coupons to stimulate more consumers, provide a variety of promotion information, segment consumers, meet different needs of target consumers and gain competitive advantage in the industry is an urgent issue for businesses to pay attention to.

There are many kinds of coupons, such as hotel coupons, shopping coupons and airport coupons. The most commonly used shopping coupons are those that consume a certain amount of money to get a certain amount of coupons, and those that are issued to new customers without threshold. Research on coupons has a long history. As early as the 1980s, 
Neslin [4] pointed out that the higher the face value of coupons, the more consumers will increase their purchase amount, and the greater the purchase amount, the more likely consumers are to use coupons; Uri [5] studies the design of the face value of a single type of coupons; later, taking into account the consumer behavior, Zhang Yin [6] classifies the customers and establishes a sales volume model for different types of customers, but does not consider the issue of coupons; Xiao [7] and Zhang [8] et al. Studied the promotion methods, customers' historical behaviors and the influence of coupons on consumers' purchase intention; In recent years, many scholars [9-11] have studied the issue of enterprise coupons. Niu Yang [9] established a coupon model for the demand function from the perspective of the supply chain; Si and Yang [10] studied the targeted coupon delivery strategy under dual channels; Mu and Tang [11] studied the delivery strategy under different coupon delivery modes. In general, Stackelberg game model is used to study the competition strategy of two businesses, such as Song [12] to study the sales strategy from the perspective of channel management based on Stackelberg game. With the rise of e-coupons, research on e-coupons began to emerge [13-17]. For example, Josep Lluis [14] gives an overview of the research on e-coupons; Paul [15] studies the redemption of competitive e-coupons; Chun [16] studies the acquisition and use of restaurant mobile coupons, pointing out that restaurant aggregation has both advantages and fierce competition. Although predecessors have done a lot of relevant research in the field of coupon design, but in the competitive environment, the design of targeted marketing coupons for different customers of two businesses is still relatively rare. In this paper, we extend the literatures on coupon and examining different types of coupons for new and old customers. Also we propose a model to find the optimal design of the coupons for two businesses in competitive environment.

The remainder of the paper is organized as follows. In the next section, we propose some problem definition and formally investigate the coupon-design decisions including the coupon's face and the threshold value for different coupons in competitive environment. In the third section, we further investigate equilibrium conditions through numerical analysis. Finally, we conclude by summarizing contributions and implications.

\section{Model Formulation}

\subsection{Problem Definition}

In order to stimulate consumers' consumption, businesses usually provide different coupons for different customers. For example, for the old users with low consumption, they will push threshold reduction coupons to improve their consumption. For the new users, they will push instant reduction coupons to attract new customers. For this reason, we assume:

(1) E-coupon types

In this paper, we consider pushing threshold reduction coupons to old customers; for new users, we consider pushing instant reduction e-coupons to attract more new customers.

(2) Consumer types

According to the literature [1], when consumers actually purchase, due to the minimum consumption limit of the threshold reduction coupons launched by merchants, they can directly use the coupon when their planned purchase amount reaches the minimum consumption amount; when their planned purchase amount is lower than the minimum consumption amount, assuming that consumers are rational, there are two possibilities: One is that if the planned purchase amount of consumers is far from the minimum consumption amount, the purchase amount will not be increased, that is, the e-coupons will be abandoned; the other is that when the planned purchase amount of consumers is close to the minimum consumption amount, the purchase amount will be increased to the minimum consumption amount of e-coupons to use the coupons. For new customers, it is assumed that as long as they consume, all of them will use instant reduction e-coupons.

(3) Consumers have no financial constraints

Assuming that the consumers is not constrained by funds, once the consumers' planned purchase amount is close to the minimum consumption amount, they can add funds to meet the minimum consumption amount.

(4) Preconditions for consumers to increase the purchase amount to the minimum consumption amount

When the planned purchase amount of the consumer is close to the minimum consumption amount, if the utility of the threshold reduction e-coupon is greater than the cost of increasing the expenditure amount, the consumer will be willing to purchase more to use the threshold reduction e-coupon, that is $D_{c} \geq M-X$

Where, $D_{c}$ is the face value of the threshold reduction e-coupon, $M$ is the actual purchase amount, and $X$ is the planned purchase amount. It is estimated conservatively that if the actual purchase amount of consumers is calculated at the deduction point (in reality, it will be slightly more than the threshold point), when the planned purchase amount of consumers $X \geq D_{p}-D_{c}$, consumers are willing to add the amount to the threshold point $D_{p}$ to enjoy the preferential policy.

(5) The design of e-coupons

For the old customers, threshold reduction e-coupons are adopted, but only one threshold point is considered, that is, when the consumer consumes $D_{p}$, a coupon with face value of $D_{c}$ will be given. Multiple threshold points are not considered; for the new customers, instant reduction coupons are adopted, if the consumer's consumption exceeds the coupon's face value, the consumer only needs to pay the amount after the coupon's face value is reduced; if the consumer's consumption amount does not reach the face value of the coupon, the consumer does not need to pay any fees, and the merchant will keep the change.

In addition, under the competitive environment, in order to compete for new and old customers, the game between the two 
merchants $\mathrm{A}$ and $\mathrm{B}$, whose profit margins are close to each other and who sell similar goods, is considered. Assumptions as follows:

(6) There are $N_{A B}$ common old customers between the two businesses $\mathrm{A}$ and B. Besides, there are $N_{A}$ old customers in business $\mathrm{A}$ and $N_{B}$ old customers in business B. $N_{A}$ old customers in business $\mathrm{A}$ are allowed to register as new customers in business B at the same time. Similarly, $N_{B}$ old customers in business $\mathrm{B}$ can also register as new customers in business $\mathrm{A}$, but $N_{A B}$ old customers in both businesses will no longer be allowed to register as new customers for enjoying instant reduction coupons;

(7) It is assumed that all consumers have no preference, that is to say, they will choose the business which offers the most attractive promotion policies. The number of new customers attracted by the business is not only affected by the promotion policies of the business itself, but also by the promotion policies of the competitive business;

(8) Assume that the higher the threshold point, the greater the returned amount, i.e. the greater the coupon face value;

(9) Only consider the case of one consumption, that is, for the customers of two businesses, they may receive two threshold reduction coupons at the same time, but they will choose one which they meet the threshold point and offer a large amount of discount according to the amount they plan to purchase;

(10) For the single old customer who does not reach the threshold point in two businesses, there is no doubt that they will participate in the instant reduction promotion of another business as new customers.

The variables used in this section are shown in Table 1:

Table 1. Variables in the e-coupons design model of two merchants in competitive environment.

\begin{tabular}{|c|c|}
\hline Variables & implication \\
\hline$X_{i}$ & The planned purchase amount of each old customer of the two businesses is a random variable, $i=A, B$ \\
\hline$f_{i}(x)$ & The probability density of the planned purchase amount of the old customers $i=A, B$ \\
\hline$N_{i}$ & Number of online old customers belonging to business i only, $i=A, B$ \\
\hline$N_{A B}$ & Number of online old customers belonging to both A and B businesses \\
\hline$p_{i}$ & Unit cost ratio of two businesses, $i=A, B$ \\
\hline$Y_{i}$ & The purchase amount of each new customer of the two businesses, which is a random variable, $i=A, B$ \\
\hline$g_{i}(y)$ & Probability density of new customers' purchase amount, $i=A, B$ \\
\hline$M^{i}\left(D_{d}^{A}, D_{d}^{B}\right)$ & the number of new customers attracted by business $\mathrm{i}$, which is the function of $D_{d}^{A}$ and $D_{d}^{B} \Pi_{i}$ the profit after promotion, $i=A, B$ \\
\hline \multicolumn{2}{|c|}{ Decision variables } \\
\hline$D_{p}^{i}$ & the threshold point of business $\mathrm{i}, i=A, B$ \\
\hline$D_{d}^{i}$ & the face value of the instant reduction e-coupons provided by business i, $i=A, B$ \\
\hline
\end{tabular}

\subsection{Model Formulation}

First, consider the profit of business A:

The profit of the business $\mathrm{A}$ is composed of the basic profit $\Pi_{0}^{A}$ before the promotion and the profit brought by the promotion.

the basic profit:

$$
\Pi_{0}^{A}=\int_{0}^{\max 1}\left(x-p_{A} x\right) f_{A}(x) d x \times\left(N_{A}+N_{A B}\right),
$$

Then consider the profit due to promotions:

1) First of all, consider the old customers only belonging to business $\mathrm{A}$, according to the assumption (8) and (9),

For the $N_{A} \times \int_{0}^{D_{p}^{A}-D_{c}^{A}} f_{A}(x) d x$ old customers who do not reach the threshold point and are far away the threshold point, they will transfer to business $\mathrm{B}$ to enjoy the instant reduction coupons of new customers, so business A will lose the profits brought by the consumption of these customers;

For the $N_{A} \times \int_{D_{p}^{A}-D_{c}^{A}}^{D_{p}^{A}} f_{A}(x) d x$ old customers, there are two possibilities for those people who are close to the threshold point but fail to reach the threshold point. When $X_{1}-\left(D_{p}^{A}-D_{c}^{A}\right)>D_{d}^{B}$, the amount of consumption is closer to the threshold point, they will choose to enjoy the threshold reduction coupons provided by business A; on the contrary, when $X-\left(D_{p}^{A}-D_{c}^{A}\right)<D_{d}^{B}$, consumers will turn to business B as new customers;

For the $N_{A} \int_{D_{p}^{A}}^{\max f^{\prime}} f_{A}(x) d x$ old customers whose consumption is more than threshold points, there is no doubt that they will choose to enjoy the threshold reduction coupons provided by business A.

Therefore, the profit of this part is 


$$
\begin{aligned}
\Delta \Pi_{N_{A}}= & -\left[N_{A} \times \int_{0}^{D_{p}^{A}-D_{c}^{A}} f_{A}(x) d x \times \int_{0}^{D_{p}^{A}-D_{c}^{A}}\left(1-p_{A}\right) \times x \times f_{A}(x) d x\right] \\
& -\left[N_{A} \times \int_{D_{p}^{A}-D_{c}^{A}}^{D_{p}^{A}-D_{c}^{A}+D_{d}^{B}} f_{A}(x) d x \times \int_{D_{p}^{A}-D_{c}^{A}}^{D_{p}^{A}-D_{c}^{A}+D_{d}^{B}}\left(1-p_{A}\right) \times x \times f_{A}(x) d x\right] \times I_{\left\{0<D_{c}^{A}<D_{d}^{B}\right\}} \\
& +\left[\int_{D_{p}^{A}-D_{c}^{A}+D_{d}^{B}}^{D_{p}^{A}}\left(1-p_{A}\right)\left(D_{p}^{A}-x\right) f_{A}(x) d x-D_{c}^{A}\right] \times N_{A} \times \int_{D_{p}^{A}-D_{c}^{A}+D_{d}^{B}}^{D_{p}^{A}} f_{A}(x) d x \times I_{\left\{0<D_{d}^{B}<D_{c}^{A}\right\}} \\
& -D_{c}^{A} \times N_{A} \times \int_{D_{p}^{A}}^{\max 1^{\prime}} f_{A}(x) d x
\end{aligned}
$$

2) Second, consider the old customers who belong to both business A and B. According to the assumption (8) and (9),

For the old customers who belong to both businesses A and $\mathrm{B}$, they can compare the promotion policy of business A and B and choose the most suitable businesses for their consumption according to their planned consumption amount.

For the old customers with the consumption amount between 0 and $D_{p}^{A}-D_{c}^{A}$, there are two possibilities. When $D_{p}^{B}<D_{p}^{A}-D_{c}^{A}$, the old customers with the consumption amount between $D_{p}^{B}$ and $D_{p}^{A}-D_{c}^{A}$ will choose to use the threshold reduction e-coupon provided by business $\mathrm{B}$, and for business $\mathrm{A}$, the original profits of this part of the consumption will be lost; the old customers with the consumption amount between 0 and $D_{p}^{B}$ will still not enjoy any discount, so there will be no change in profits; when $D_{p}^{B}>D_{p}^{A}-D_{c}^{A}$, the old customers with the consumption amount between 0 and $D_{p}^{A}-D_{c}^{A}$ will still not enjoy any discounts either;

For those people whose consumption amount is close to the threshold point, but fails to reach the threshold point, when $X_{A}-\left(D_{p}^{A}-D_{c}^{A}\right)>D_{c}^{B}$, the customer will choose to enjoy the threshold reduction coupon provided by merchant $\mathrm{A}$ whether the threshold reduction point $D_{p}^{B}$ of merchant $\mathrm{B}$ is satisfied $D_{p}^{B}<D_{p}^{A}-D_{c}^{A}<D_{p}^{A}$ or $D_{p}^{A}-D_{c}^{A}<D_{p}^{B}<D_{p}^{A}$; otherwise, if $\left(D_{p}^{A}-D_{c}^{A}\right) \leq X_{A}<\left(D_{p}^{A}-D_{c}^{A}\right)+D_{c}^{B} \quad$ so $\quad$ when $D_{p}^{B} \leq D_{p}^{A}-D_{c}^{A}<D_{p}^{A}$, the customer will choose the threshold reduction coupon provided by merchant $\mathrm{B}$; When $D_{p}^{A}-D_{c}^{A}<D_{p}^{B}<D_{p}^{A}$, due to the preferential policies of merchant $\mathrm{A}$, so it is natural for customer to choose the threshold reduction coupon provided by merchant A;

When $D_{p}^{B}<D_{p}^{A}$, the customers with consumption amount above the threshold reduction point $D_{p}^{A}$ will obviously choose the threshold reduction coupons provided by business A; when $D_{p}^{B}>D_{p}^{A}$, the customers with consumption amount between $D_{p}^{A}$ and $D_{p}^{B}$ will still choose the threshold reduction coupons provided by business $A$, but if the customers with consumption amount above $D_{p}^{B}$ will choose the threshold reduction coupons provided by business $\mathrm{B}$, business A will lose the profits of this part;

Therefore, the profit changes $\Delta \Pi_{N_{A B}}^{A}$ brought by the customers who belongs to the businesses $\mathrm{A}$ and $\mathrm{B}$ are as follows:

$$
\begin{aligned}
\Delta \Pi_{A B}^{A}= & -N_{A B} \times \int_{D_{p}^{B}}^{D_{p}^{A}-D_{c}^{A}} f_{A}(x) d x \times \int_{D_{p}^{B}}^{D_{p}^{A}-D_{c}^{A}}\left(1-p_{A}\right) x f_{A}(x) d x \times I_{\left\{D_{p}^{B}<D_{p}^{A}-D_{c}^{A}\right\}}+N_{A B} \times \int_{D_{p}^{A}-D_{c}^{A}+D_{c}^{B}}^{D_{p}^{A}} f_{A}(x) d x \times\left[\int_{D_{p}^{A}-D_{c}^{A}+D_{c}^{B}}^{D_{p}^{A}}\left(1-p_{A}\right)\left(D_{p}^{A}-x\right) f_{A}(x) d x-D_{c}^{A}\right] \times I_{\left\{D_{p}^{B}<D_{p}^{A}-D_{c}^{A}<D_{p}^{A}\right\}} \\
& -N_{A B} \times \int_{D_{p}^{A}-D_{c}^{A}+D_{c}^{B}}^{D_{p}^{A}} f_{A}(x) d x \times\left[\int_{D_{p}^{A}-D_{c}^{A}}^{D_{p}^{A}-D_{c}^{A}+D_{c}^{B}}\left(1-p_{A}\right) x f_{A}(x) d x\right] \times I_{\left\{D_{p}^{B} \leq D_{p}^{A}-D_{c}^{A}<D_{p}^{A}\right\}}+N_{A B} \times \int_{D_{p}^{A}-D_{c}^{A}}^{A} f_{A}(x) d x \times\left[\int_{D_{p}^{A}-D_{c}^{A}}^{A}\left(1-p_{A}^{A}\right)\left(D_{p}^{A}-x\right) f_{A}(x) d x-D_{c}^{A}\right] \times I_{\left\{D_{p}^{A}-D_{c}^{A}<D_{p}^{B} \leq D_{p}^{A}\right\}} \\
& +N_{A B} \times \int_{D_{p}^{A}-D_{c}^{A}}^{D_{p}^{A}} f_{A}(x) d x \times\left[\int_{D_{p}^{A}-D_{c}^{A}}^{D_{p}^{A}}\left(1-p_{A}\right)\left(D_{p}^{A}-x\right) f_{A}(x) d x-D_{c}^{A}\right] \times I_{\left\{D_{p}^{A}-D_{c}^{A}<D_{p}^{A} \leq D_{p}^{B}\right\}} \\
& +N_{A B} \times \int_{D_{p}^{A}}^{\max f_{A}^{\prime}} f_{A}(x) d x \times\left[-D_{c}^{A}\right] \times I_{\left\{D_{p}^{B} \leq D_{p}^{A}\right\}}+N_{A B} \times \int_{D_{p}^{A}}^{D_{p}^{B}} f_{A}(x) d x \times\left[-D_{c}^{A}\right] \times I_{\left\{D_{p}^{A}<D_{p}^{B}\right\}} \\
& -N_{A B} \times \int_{D_{p}^{B}}^{\max f_{A}^{\prime}} f_{A}(x) d x \times\left[\int_{D_{p}^{B}}^{\max x^{\prime}}\left(1-p_{A}\right) x f_{A}(x) d x\right] \times I_{\left\{D_{p}^{A}<D_{p}^{B}\right\}}
\end{aligned}
$$

3) Finally, consider the new customers transferred from the business $\mathrm{B}$ and the new customers attracted

It can be similarly obtained from (1) that for the old customers of business B who do not reach and are far away 
from the threshold reduction point, they will turn to business A to enjoy the instant reduction coupons of new customers, and for those who are close to the threshold reduction point but do not reach the threshold reduction point, when
$X_{B}-\left(D_{p}^{B}-D_{c}^{B}\right)<D_{d}^{A}$, consumers will turn to business A as new customers, and the benefits are as follows respectively:

$$
\begin{aligned}
& \int_{0}^{D_{d}^{A}}-p_{A} y g_{A}(y) d y \times N_{B} \times \int_{0}^{D_{p}^{B}-D_{c}^{B}} f_{B}(x) d x \times \int_{0}^{D_{d}^{A}} g_{A}(y) d y \\
& +\left[\int_{D_{d}^{A}}^{\max 2}\left(y-D_{d}^{A}-p_{A} y\right) g_{A}(y) d y\right] \times N_{B} \times \int_{0}^{D_{p}^{B}-D_{c}^{B}} f_{B}(x) d x \times \int_{D_{d}^{A}}^{\max 2} g_{A}(y) d y
\end{aligned}
$$

and

$$
\begin{aligned}
& \int_{0}^{D_{d}^{A}}-p_{A} y g_{A}(y) d y \times N_{B} \times \int_{D_{p}^{B}-D_{c}^{B}}^{D_{p}^{B}-D_{c}^{B}+D_{d}^{A}} f_{B}(x) d x \times \int_{0}^{D_{d}^{A}} g_{A}(y) d y \\
& +\left[\int_{D_{d}^{A}}^{\max 2}\left(\mathrm{y}-D_{d}^{A}-p_{A} y\right) g_{A}(y) d y\right] \times N_{B} \times \int_{D_{p}^{B}-D_{c}^{B}}^{D_{p}^{B}-D_{c}^{B}+D_{d}^{A}} f_{B}(x) d x \times \int_{D_{d}^{A}}^{\max 2} g_{A}(y) d y
\end{aligned}
$$

In addition, due to the preferential strength of business A, new customers are attracted, so their benefits are as follows:

$$
\begin{aligned}
& \int_{0}^{D_{d}^{A}}-p_{A} y g_{A}(y) d y \times M^{A}\left(D_{d}^{A}, D_{d}^{B}\right) \times \int_{0}^{D_{d}^{A}} g_{A}(y) d y \\
& +\left[\int_{D_{d}^{A}}^{\max 2}\left(y-D_{d}^{A}-p_{A} y\right) y g_{A}(y) d y\right] \times M^{A}\left(D_{d}^{A}, D_{d}^{B}\right) \times \int_{D_{d}^{A}}^{\max 2} g_{A}(y) d y
\end{aligned}
$$

Therefore, the profits $\Pi_{\text {new }}^{A}$ generated by new customers attracted by business A is

$$
\begin{aligned}
\Pi_{n e w}^{A}= & \int_{0}^{D_{d}^{A}}-p_{A} y g_{A}(y) d y \times N_{B} \times \int_{0}^{D_{p}^{B}-D_{c}^{B}} f_{B}(x) d x \times \int_{0}^{D_{d}^{A}} g_{A}(y) d y \\
& +\left[\int_{D_{d}^{A}}^{\max 2}\left(y-D_{d}^{A}-p_{A} y\right) g_{A}(y) d y\right] \times N_{B} \times \int_{0}^{D_{p}^{B}-D_{c}^{B}} f_{B}(x) d x \times \int_{D_{d}^{A}}^{\max 2} g_{A}(y) d y \\
& +\int_{0}^{D_{d}^{A}}-p_{A} y g_{A}(y) d y \times N_{B} \times \int_{D_{p}^{B}-D_{c}^{B}}^{D_{p}^{B}-D_{c}^{B}+D_{d}^{A}} f_{B}(x) d x \times \int_{0}^{D_{d}^{A}} g_{A}(y) d y \\
& +\left[\int_{D_{d}^{A}}^{\max ^{A} 2}\left(\mathrm{y}-D_{d}^{A}-p_{A} y\right) g_{A}(y) d y\right] \times N_{B} \times \int_{D_{p}^{B}-D_{c}^{B}+D_{d}^{A}}^{D_{D}^{B}-D_{c}^{B}} f_{B}(x) d x \times \int_{D_{d}^{A}}^{\max ^{A}} g_{A}(y) d y \\
& +\int_{0}^{D_{d}^{A}}-p_{A} y g_{A}(y) d y \times M^{A}\left(D_{d}^{A}, D_{d}^{B}\right) \times \int_{0}^{D_{d}^{A}} g_{A}(y) d y \\
& +\left[\int_{D_{d}^{A}}^{\max ^{A} 2}\left(y-D_{d}^{A}-p_{A} y\right) y g_{A}(y) d y\right] \times M^{A}\left(D_{d}^{A}, D_{d}^{B}\right) \times \int_{D_{d}^{A}}^{m_{A}} g_{A}(y) d y
\end{aligned}
$$

All in all, the final revenue $\Pi_{A}$ generated by the promotion of business $\mathrm{A}$ is

$$
\Pi_{A}=\Pi_{0}^{A}+\Delta \Pi_{N_{A}}+\Delta \Pi_{N_{A B}}^{A}+\Pi_{n e w}^{A}
$$

Similarly, we can get the revenue $\Pi_{B}$ generated by the promotion of business B as follows

$$
\Pi_{B}=\Pi_{0}^{B}+\Delta \Pi_{N_{B}}+\Delta \Pi_{N_{A B}}^{B}+\Pi_{\text {new }}^{B}
$$


The specific expression is similar to that of business A, so it will not be repeated here.

In this way, for the information of business $A$ and $B$ is symmetrical, they can fully observe each other's decision-making behavior. The goal of the two businesses is to choose the right $D_{p}^{i}, D_{c}^{i}$ and ${ }_{D_{d}^{i}}$ to maximize their own benefits, that is

$$
\max \Pi_{A}=\Pi_{0}^{A}+\Delta \Pi_{N_{A}}+\Delta \Pi_{N_{A B}}^{A}+\Pi_{n e w}^{A}
$$

And

$$
\max \Pi_{B}=\Pi_{0}^{B}+\Delta \Pi_{N_{B}}+\Delta \Pi_{N_{A B}}^{B}+\Pi_{\text {new }}^{B}
$$

\subsection{Model Solution}

Because this is a very complex game theory problem with three decision variables, we cannot find its numerical solution, but we can combine simulation analysis, based on Stackelberg game for reverse reasoning. Given the decision-making structure of first mover merchants, the follower merchants make decisions, thus forming the optimal decision-making set of follower merchants to first mover merchants' behaviors; then it is transformed into the decision-making problem of first mover merchants. Given the optimal decision-making set of follower merchants, first mover merchants determine their choice to maximize their own profits. This will goes on and on until one side can't go on. The algorithm steps are as follows:

1) Suppose business A (first mover merchant) takes the lead in initiating the offer and sets its threshold reduction point as $D_{p}^{A}$, its coupon combination as $\left(D_{p}^{A}, D_{c}^{A}, D_{d}^{A}\right)$ will be obtained according to the optimal strategy of a single merchant's coupon (introduced in another article);

2) According to the promotion strategy of business A and the average consumption of its old customers, business B sets the initial threshold reduction point $D_{p 0}^{B}$ and also obtains the optimal preferential combinations $\left(D_{p 0}^{B}, D_{c 0}^{B}\right.$, $\left.D_{d 0}^{B}\right)$ from the optimal strategy of a single merchant's coupons, then calculates the corresponding profits $\Pi_{0}^{B}$ according to formula (2);

3 ) Then, on the basis of the initial value $D_{p 0}^{B}$, the threshold reduction point $D_{p}^{B}$ is incremented in steps of $\Delta=10$ yuan, and steps (2) are repeated to obtain the optimal preferential combination $\left(D_{p i}^{B}, D_{c i}^{B}, D_{d i}^{B}\right)$ and corresponding profit $\Pi_{i}^{B}$ in turn;

4) Repeat step (3) until the upper bound of the threshold reduction point set by business $\mathrm{B}$ is reached, and compare the revenue $\Pi_{0}^{B}$ with each revenue $\Pi_{i}^{B}(\mathrm{i}=1,2$, $3 \ldots .$.$) , find out the maximum value \Pi^{\mathrm{B}^{*}}$ and the corresponding preferential combination $\left(D_{p}^{B^{*}}, D_{c}^{B^{*}}, D_{d}^{B^{*}}\right)$ which is the best decision made by business $\mathrm{B}$ for business A;

5) On the contrary, according to the preferential policies of business B, business A will repeat steps (2) - (4) to get the optimal decision-making combination $\left(D_{p}^{A^{*}}, D_{c}^{A^{*}}\right.$, $D_{d}^{A^{*}}$;

6) Business A and B repeat this until one of them is unable to continue due to the loss of profits and exits the game.

\section{Numerical Analysis}

Suppose business A and B sell the same type of products. Considering that they are in a completely competitive environment, when business A initiates a promotion, merchant $\mathrm{B}$ will respond accordingly. Let the unit cost ratio of business $\mathrm{A}$ is $p_{A}=0.6$, the unit cost ratio of business $\mathrm{B}$ is $p_{B}=0.4$, that is, the unit profit of business $\mathrm{B}$ is larger than that of business A. Suppose the per capita consumption amount $X_{A}$ of business A follows the normal distribution, that is $X_{A} \sim \mathrm{N}\left(80,20^{2}\right)$, and the per capita consumption amount of business B $X_{B} \sim \mathrm{N}\left(100,50^{2}\right)$. Suppose that the number of old customers which is only belong to the business A is 1350 , that is $N_{A}=1350$, and its maximum consumption amount Max $1=800$ yuan. Suppose that the number of old customers which is only belong to the business $\mathrm{B}$ is 860 , that is $N_{B}=860$, and its maximum consumption amount $\max 2=$ 1200. The common customers of both businesses A and B is 200 , that is $N_{A B}=200$.

Since the number of new customers is not only affected by the face value of their own discount coupons, but also affected by the face value of their opponents' discount coupons, it is assumed that the number of new customers of business $\mathrm{A}$ is a function as

$$
M^{A}\left(D_{d}^{A}, D_{d}^{B}\right)=k_{1} \log _{10} D_{d}^{A}-k_{2} \log _{10} D_{d}^{B}=1000 \log _{10} D_{d}^{A}-100 \log _{10} D_{d}^{B}
$$

Assume that the function of the number of new customers of business B is

$$
M^{B}\left(D_{d}^{B}, D_{d}^{A}\right)=k_{1}^{\prime} \log _{10} D_{d}^{B}-k_{2}^{\prime} \log _{10} D_{d}^{A}=800 \log _{10} D_{d}^{B}-120 \log _{10} D_{d}^{A}
$$

Assume that the consumption amount of each new customer follows a normal distribution as,

$$
Y \sim f_{i}(\mathrm{y})=\frac{1}{\sqrt{2 \pi} \times \sigma_{i}} e^{-\frac{\left(y-\frac{D_{d i}}{1-p_{i}}\right)^{2}}{2 \times \sigma_{i}^{2}}}, y \in(-\infty,+\infty), \quad i=A, B
$$

Suppose business A starts a promotion and adopts the promotion strategy as $D_{p}^{A}=100, D_{c}^{A}=20, D_{d}^{A}=10$. so business B looks for its own optimal preferential combination according to the action of business A. In order to simplify the calculation, suppose that the threshold point $D_{p}^{B}$ is increased by 10 yuan between $(0,1000]$. Under one certain $D_{p i}^{B}$, the optimal combination $\left(D_{p i}^{B}, D_{c i}^{B}, D_{d i}^{B}\right)$ is found, and the corresponding revenue $\Pi_{i}^{B}$ is obtained according to formula 
(2), and the final combination $\left(D_{p}^{B^{*}}, D_{c}^{B^{*}}, D_{d}^{B^{*}}\right)$ with the highest return value of business $B$ is obtained according to step (4). Using MATLAB and step-by-step search, table 2 is obtained as follows:

Table 2. Optimal coupon design scheme between business $A$ and B in each period.

\begin{tabular}{lllllllll}
\hline & \multicolumn{7}{c}{ Business A } & \multicolumn{7}{c}{ Business B } \\
\cline { 2 - 9 } & $D_{p}^{A}$ & $D_{c}^{A}$ & $D_{d}^{A}$ & $\Pi_{A}$ & $D_{p}^{B}$ & $D_{c}^{B}$ & $D_{d}^{B}$ & $\Pi_{B}$ \\
\hline 1 & 100 & 18 & 6 & 46718.3 & --- & --- & --- & --- \\
2 & --- & --- & --- & -- & 100 & 18.65 & 15.05 & 80367.5 \\
3 & 100 & 20.02 & 20.16 & 33602.8 & -- & --- & -- & -- \\
4 & --- & --- & --- & --- & 80 & 28.10 & 26.39 & 65351.9 \\
\hline
\end{tabular}

It can be seen from table 2 that under the condition that no competitor exists, business A obtains the optimal coupon delivery strategy when the threshold reduction point is set to 100: Deduct 18 yuan when consumption amount reach 100 yuan for the old customers, deduct 6 yuan for the new customers when they are shopping, and the profit is 46718.3 yuan;

At this time, if business B participates in the competition, in order not to lose the old customers, and strive for new customers as much as possible. Therefore, business B's strategy is to compete with business A, preferring to lose money on the old customers, business A will put the face value slightly higher than that of business $\mathrm{A}$, and at the same time enlarge the face value of the coupon for new customers to 15.05 yuan, so that the customers belonging to both businesses A and B will choose business B to consume for their own interests;

In the face of competition from business $\mathrm{B}$, business $\mathrm{A}$ adjusted its coupon delivery strategy: the preferential quota for old customers was increased to 20.02 yuan, while the preferential quota for new customers was also increased to 20.16 yuan, which would bring the customers originally belonging to business $\mathrm{B}$ to join as new customers of business $\mathrm{A}$, but its revenue would be much lower than the original, down to only 33602.8 yuan.

In the case of changes in the competitive environment, in order to retain the old customers, business $\mathrm{B}$ reduces the threshold for the old customers and its promotion strategy is changed as deduct 28.10 yuan when its consumption reach 80 yuan, and then increases the preferential quota for new customers to 26.39 yuan. At this time, the revenue of business $\mathrm{B}$ will also decrease to 65351.9. Till now, the calculation will be stopped because there is no new preferential strategy for business A, which means that business A can no longer participate in the competition. The above also explains that some big businesses with high profits in real life often use this vicious competition strategy to defeat competitors, such as the previous didi taxi case.

\section{Conclusion}

Different types of coupons are designed according to the new and old customers in this paper. For the old customers, the threshold reduction coupons are used; for the new customers, the instant reduction coupons are used. And then based on the classification of consumer behavior, in the view of merchants, a coupon model for new and old customers of two merchants is built in the competitive market environment. Finally taking two merchants with different profit as an example, the influence of competitors on the coupon delivery strategy of merchants is analyzed. The results show that the existence of competitors will force the merchants to cut profit in favor of the customers and enlarge the coupon's face value to the maximum value they can bear.

In this paper, the coupon design strategy of two merchants in the competitive environment is studied. In the future, the coupon design strategy model of more than two merchants or on the premise of consumer preference can be further studied.

\section{Acknowledgements}

The authors wish to thank the Jiangsu Social Science Foundation for their financial support during the early stages of this project and Kai Chen, a graduate student of Nanjing University of Science and Technology, for the help in numerical calculation.

\section{References}

[1] Lu Song, Research on the strategy of e-coupon delivery based on consumer behavior [D], Master Dissertation of Nanjing University of technology 2018.

[2] Yanqiu Liu, Huina Yue, The model and solution of coupon promotion pricing $[\mathrm{J}]$, Journal of Shenyang University of Technology, Vol. 36 No. 2, 2014.

[3] Yinyuan Si, Wensheng Yang, Discrimination pricing and Strategy of competitive enterprises considering consumer transfer cost and coupons [J], Enterprise economy, Issue 8, 2018: 111-118.

[4] Neslin S A, Henderson C, Quelch J. Consumer promotions and the acceleration of product purchase [J]. Marketing Science, 1985, 4 (2): 147-166.

[5] Uri Ben-Zion, Aharon Hibshoosh and Uriel Spiegel, The Optimal Face Value of a Discount Coupon [J], Journal of Economics and Business, 1999, 51: 159-174.

[6] Zhang Ying, Hao Yi-ge, Wang Yan-hua. Pricing model with coupon selling and its solution [J]. Journal of Shenyang University of Technology, 2005, 27 (6): 691-694.

[7] Yumeng Xiao, A study on the influence of the promotion of takeout on consumers' purchase intention [J], Commercial economy, Issue 2, 2018: 57-58.

[8] Jiantong Zhang, Chencheng Fang, The Effects of Historic Behaviors and E - coupon Promotion on Consumer Purchase Decision-Based on an Experimental Study [J], Soft science, Feb. 2017, Vol. 31, No. 2: 109-112.

[9] Niu Yang. The research about coupon distributed mode and pricing of enterprise supply chain and coordination strategy [D]. Chengdu: University of Electronic Science and Technology of China, 2010: 20-23. 
[10] Yinyuan Si, Wensheng Yang etc. Research on the strategy of targeted coupon delivery under the dual channel model [J/OL], Industrial Engineering and management, http://kns.cnki.net/kcms/detail/31.1738.T.20190509.1315.026. html.

[11] Yinping Mu, Xiaowo Tang, Yang Niu, Pricing and coordination strategies of supply chain under different discount coupon issuing modes [J], Chinese Management Science, 2011, 19 (6): 48-56.

[12] Fengsen Song, Research on sales strategy from the perspective of channel management -- the optimal pricing model based on Stackelberg game $[\mathrm{J}]$, Operation and management, issue 2, 2019: 135-138.

[13] Mikhael Shor, Richard L. Oliver, Price discrimination through online couponing: Impact on likelihood of purchase and profitability [J], Journal of Economic Psychology, 27 (2006): 423-440.

[14] Josep-Lluis Ferrer-Gomila, M. Francisca Hinarejos, Llorenc Huguet-Rotger, A survey on electronic coupons [J], Computers \& Security, 77 (2018): 106-127.

[15] Paul Mills, Cesar Zamudio, Scanning for discounts: examining the redemption of competing mobile coupons [J], Journal of the Academy of Marketing Science, (2018) 46: 964-982.

[16] Chun Qiu, Ping Zhao, Mobile coupon acquisition and redemption for restaurants: The effects of store clusters as a double-edged sword [J], Journal of Business Research, 103 (2019) 163-172. 